\title{
An IL8-CEA Combined Score is Stage-Specific in Colorectal Cancer
}

\author{
Ashok Gunawardene ${ }^{1 *}$, Stephanie Manning ${ }^{1}$, Ana Holley ${ }^{1}$, Gisela Kristono ${ }^{1}$, Kirsty Danielson ${ }^{1}$, \\ Ali Shekouh ${ }^{2}$, Peter Larsen ${ }^{1}$, Elizabeth Dennett ${ }^{1,2}$
}

\author{
*Corresponding author: \\ Ashok Gunawardene, MD \\ Department of Surgery \& Anaesthesia \\ University of Otago \\ 23 Mein Street, Newtown \\ Wellington South 6021, New Zealand \\ E-mail: ARG240@outlook.com \\ Tel: +64 43855541 \\ ORCID Number: 0000-0003-1032-3455
}

\author{
'Department of Surgery \& Anaesthesia, University of Otago, Wellington, New Zealand \\ ${ }^{2}$ Capital \& Coast District Health Board, Wellington, New Zealand
}

\section{ABSTRACT}

Background: A raised pre-treatment carcinoembryonic antigen (CEA) level is frequently observed in colorectal cancer patients with metastatic disease and is thought to be a reflection of disease burden. Additionally, there is a growing body of evidence highlighting the influence of a pro-inflammatory host-response on colorectal cancer progression. Proinflammatory cytokines IL- $1 \beta$, IL- 6 , IL-8 and TNF $\alpha$ have been implicated in this relationship through experimental and clinical studies. We set out to evaluate whether combining circulating inflammatory markers, such as these, with CEA improves upon the stagespecificity of CEA alone.

Methods: Sixty patients were enrolled prospectively at a single centre over a sixteen month period into three groups: control $(n=19)$, stage II $(n=22)$ and stage IV $(n=19)$ colorectal cancer. Baseline levels of carcinoembryonic antigen (CEA) as well as plasma IL-1 $\beta, \mathrm{IL}-6$, IL-8 and TNF $\alpha$ were compared across the groups. The ability of each marker to distinguish patients by disease status was evaluated using Receiver Operator Characteristic (ROC) curve and Chi-squared analysis.

Results: CEA, IL-6 and IL-8 increased significantly between the three groups. CEA and IL-8 were most effective in discriminating between stage II and stage IV disease groups. We found IL8-CEA score was more effective in discriminating between stage II and stage IV CRC (OR 10.3, 95\% $\mathrm{Cl} 1.1-92.2, \mathrm{p}=0.01$ ) compared with CEA alone (OR 5.4, 95\% $\mathrm{Cl} 1.3-27.8$, $\mathrm{p}=0.01)$.

Conclusion: Combining IL-8 with CEA improved upon the stage-specificity of CEA alone. This suggests a potential value for combining circulating inflammatory biomarkers with CEA to accurately stage CRC.

Key words: IL-8, CEA, colorectal cancer

\section{INTRODUCTION}

Colorectal cancer (CRC) is the third largest contributor to the global cancer burden and, despite advances in treatment, it remains a major source of cancerrelated mortality (1). At presentation, approximately $20 \%$ of patients have distant metastasis (2) and experience significantly poorer outcomes as a result. Therefore, wherever possible, patients are routinely screened for distant metastases promptly after initial diagnosis. This is often pivotal in selecting the appropriate management strategy, which may involve assessment of resectability, or the decision of whether or not to pursue radical treatment. 
Carcinoembryonic antigen (CEA) is a widely used tumour marker in colorectal cancer and raised circulating levels are frequently observed in patients with metastatic disease $(3,4)$. This suggests a relationship between CEA and tumour burden, leading some to advocate its integration into the TNM staging system (5-7). Circulating biomarkers, such as CEA, have the advantage of being obtained at minimal discomfort or inconvenience to the patient. Furthermore, results may be available at the outset, in contrast to some elements of the TNM staging system, such as tumour depth, which in turn may require assessment of a surgical specimen.

As the influence of inflammation on colorectal cancer progression becomes better understood, so does the importance of the host's immune and inflammatory response to cancer (8-10). Experimental evidence points to a bidirectional relationship between tumour and host cells, both within its microenvironment and at distant sites, that is integral to the development of metastatic disease $(11,12)$. IL-1 13, IL-6, IL-8 and TNF $\alpha$ are among the inflammatory mediators most consistently implicated in this process by promoting endothelial-mesenchymal transition, angiogenesis and chemo-attraction of neutrophils at metastatic sites (12-16). Like CEA, these inflammatory markers have been observed to increase with advancing disease stage $(17,18)$. Additionally, indicators of systemic inflammation such as neutrophil lymphocyte ratio (NLR) have been combined with CEA to improve on its diagnostic ability $(19,20)$. However, very little is known as to whether combining circulating inflammatory biomarkers with CEA adds value in evaluating colorectal cancer progression. Therefore, the aim of this study was to determine whether combining circulating inflammatory cytokines with CEA improves upon the disease stage-specificity of CEA alone.

\section{METHODS}

\section{Patient selection}

Patients were recruited prospectively at a single metropolitan centre over a sixteen-month period between October 2016 and February 2018.

Healthy control patients were enrolled immediately prior to undergoing a colonoscopy requested on the suspicion of lower gastrointestinal malignancy. Patients were excluded if any evidence of inflammation or malignancy was subsequently detected. Patients were recruited into the stage II group on the basis of pretreatment radiological staging. Rectal cancer patients underwent pre-operative staging MRI pelvis and CT chest abdomen and pelvis, and colon cancer patients underwent pre-operative CT chest, abdomen and pelvis. Imaging was reviewed by two Consultant Radiologists. Staging was confirmed postoperatively on pathology in the case of colon cancer patients. This was not applied to patients with rectal cancer due to a proportion undergoing neo-adjuvant therapy. Consecutive patients presenting with metastatic colorectal cancer at diagnosis were recruited into the stage IV group. Disease stages were assigned according to the $8^{\text {th }}$ edition of American Joint Committee on Cancer guidance (21).

Exclusion criteria were: age below 18 years, emergency presentation, chronic inflammatory conditions including inflammatory bowel disease, other synchronous primary malignancy, colorectal cancer other than adenocarcinoma, and the use of immunosuppressive therapy including oral corticosteroids either at the time of, or within three months prior to, sample withdrawal.

All patients recruited into this study gave their written informed consent and ethics approval was granted by the Health and Disability Ethics Committee (HDEC ref: 18/CEN/138).

\section{Plasma sample collection and storage}

A twenty millilitre venous blood specimen was withdrawn using a 23G BDTM vacutainer blood collection set into sodium citrate vacutainer tubes after discarding the first $5 \mathrm{~mL}$. Wherever possible, venepuncture was performed from the antecubital fossa following a standard protocol and samples were centrifuged within 30 minutes of withdrawal at $2000 \times \mathrm{g}$ for ten minutes and plasma was stored at -80 degrees Celsius until time of analysis.

\section{Cytokine assays}

All assays were performed within twenty-four months of sample collection and in two or fewer freezethaw cycles. Levels of IL-1 $\beta$, IL- 6, IL- 8 and TNF $\alpha$ were quantified from plasma using commercially available sandwich ELISA kits specific for each cytokine (Invitrogen, ThermoFisher Scientific) as per the manufacturers instructions. Absorbances were measured using the "Multiskan GO" (Thermo Scientific) specialised spectrophotometer. The detection limits were IL-6: 2-200 pg/mL, IL-1B: 2-150 pg/mL, IL-8: 2-250 pg/mL and TNFa: $4-500 \mathrm{pg} / \mathrm{mL}$. Analytes with a detection rate below $75 \%$ were excluded. CEA (Roche Cobas e601, reference range $<3.5 \mu \mathrm{g} / \mathrm{L}$ ) was measured at the clinical diagnostics laboratory of the host institution. 


\section{Statistical analysis}

Statistical packages SPSS ${ }^{\circledR}$ version $24.0\left(\right.$ IBM $\left.^{\circledR}\right)$ and GraphPad Prism 7 (San Diego, CA, USA) were used for analysis. The Shapiro-Wilk test of normality was used to distinguish parametric from non-parametric distributions. Continuous variables were presented as mean \pm standard deviation (SD) for parametric data and median (interquartile range, IQR) for non-parametric. The groups were compared using ANOVA for parametric and the Kruskal-Wallis test for nonparametric variables. Receiver Operator Characteristic (ROC) curve analysis was performed to evaluate ability of markers to differentiate between patient groups and select the appropriate cut-off value based on optimal sensitivity and specificity. For categorical variables, proportions were compared between groups using Chi squared test. A $p$ value of $\leq 0.05$ was taken as the level of statistical significance.

\section{RESULTS}

A total of sixty patients were included in the study across three groups: control $(n=19)$, stage II $(n=22)$ and stage IV ( $n=19)$. The mean age was 65.9 years (range 29-85) with 33 (55.0\%) males and 27 (45.0\%) females. By ethnicity, 53 (88.3\%) were European, 4 (6.7\%) Maori and $3(5.0 \%)$ other. The median body mass index (BMI) was $28.2 \mathrm{~kg} / \mathrm{m}^{2}$ (IQR, 25.0-31.7). There was a lower proportion of patients with ischaemic heart disease in the stage IV group compared with controls $(p=0.05)$ and no other patient characteristics were found to vary significantly between the three groups (table 1).

IL-6 levels were detected in all and IL- 8 in 59/60 (98.3\%) patients. TNF $\alpha$ levels were detectable in $8 / 60$ (13.3\%) patients and IL-1 $\beta$ in $0 / 60$ and were therefore excluded from further analysis. When biomarker levels were compared across the three groups, CEA $(p<0.001)$, IL-6 ( $p=0.005)$ and IL-8 $(p<0.001)$ all increased significantly with disease progression (table 2 ). The increases in CEA, IL-6 and IL-8 across groups were most pronounced between stage II and stage IV (fig. 1).

\section{Control versus Stage II}

Receiver operator characteristic analyses were performed to evaluate CEA, IL- 6 and IL-8 as discriminators between patient groups. For the control versus stage II group, CEA (AUC $0.73,95 \% \mathrm{Cl} 0.58-0.89, \mathrm{p}=0.01$ ) was

Table 1 - Patient characteristics. Age is expressed as mean \pm standard deviation. BMI: body mass index, IHD: ischaemic heart disease, DM2: type two diabetes mellitus. BMI is expressed as median (interquartile range). Categorical variables are expressed as a number with the percentage of the group in brackets

\begin{tabular}{|c|c|c|c|c|}
\hline & Control & Stage II & Stage IV & $\mathrm{p}$ value \\
\hline$n$ & 19 & 22 & 19 & \\
\hline Age (years) & $68.0 \pm 12.0$ & $66 \pm 12.0$ & $63.0 \pm 12.0$ & 0.45 \\
\hline $\begin{array}{l}\text { Gender } \\
\text { Male } \\
\text { Female }\end{array}$ & $\begin{array}{c}7(36.8) \\
12(63.2)\end{array}$ & $\begin{array}{c}15(68.2) \\
7(31.8)\end{array}$ & $\begin{array}{c}11(57.9) \\
8(42.1)\end{array}$ & 0.13 \\
\hline $\begin{array}{l}\text { Ethnicity } \\
\text { European } \\
\text { Maori } \\
\text { Other }\end{array}$ & $\begin{array}{c}19(100) \\
0(0) \\
0(0)\end{array}$ & $\begin{array}{c}19(86.4) \\
2(9.1) \\
1(4.5)\end{array}$ & $\begin{array}{l}15(78.9) \\
2(10.5) \\
2(10.5)\end{array}$ & 0.34 \\
\hline $\begin{array}{l}\mathrm{BMI}, \mathrm{kg} / \mathrm{m}^{2} \\
\quad \text { Median (IQR) }\end{array}$ & $28.2(24.8-32.4)$ & $29.6(26.9-33.1)$ & $27.0(23.7-29.8)$ & 0.09 \\
\hline $\begin{array}{l}\text { Current smoker } \\
\text { IHD } \\
\text { DM2 } \\
\text { Aspirin }\end{array}$ & $\begin{array}{c}0(0) \\
4(21.1) \\
3(15.8) \\
6(31.6)\end{array}$ & $\begin{array}{c}2(9.1) \\
1(4.5) \\
3(13.6) \\
5(22.7)\end{array}$ & $\begin{array}{c}4(21.1) \\
0(0) \\
3(15.8) \\
2(10.5)\end{array}$ & $\begin{array}{l}0.10 \\
0.05 \\
0.98 \\
0.29\end{array}$ \\
\hline
\end{tabular}

Table 2 - Biomarker values by patient group. Values are expressed as median (IQR). Bolded p-values are statistically significant ( $\leq 0.05)$. IL: Interleukin; CEA: Carcinoembryonic antigen

\begin{tabular}{lcccc}
\hline & Control & Stage II & Stage IV & p value \\
\hline $\mathrm{IL}-6, \mathrm{pg} / \mathrm{mL}$ & $2.46(1.85-3.98)$ & $2.51(2.01-3.46)$ & $6.12(4.63-10.56)$ & 0.005 \\
\hdashline $\mathrm{IL}-8, \mathrm{pg} / \mathrm{mL}$ & $3.95(3.35-6.51)$ & $5.59(4.33-9.27)$ & $11.40(8.39-34.05)$ & $<0.001$ \\
$\mathrm{CEA}, \mu \mathrm{g} / \mathrm{mL}$ & $1.90(1.20-2.90)$ & $3.45(2.00-6.50)$ & $20.50(4.60-131.20)$ & $<0.001$ \\
\hline
\end{tabular}




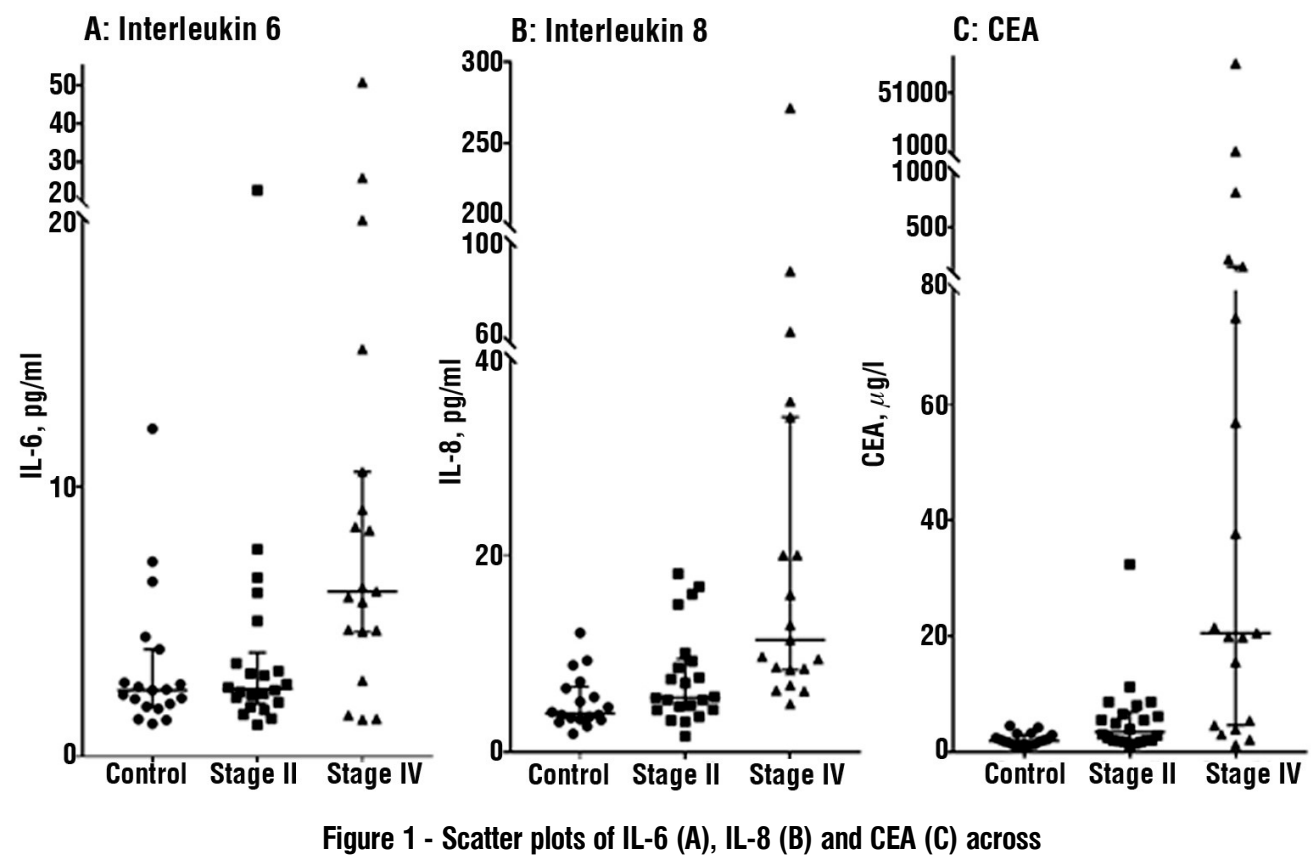

effective in differentiating between these groups in contrast to IL-6 (AUC 0.55, 95\% Cl 0.37-0.73, $\mathrm{p}=0.62$ ) or IL-8 (AUC 0.68, 95\% Cl 0.51-0.85, p=0.06) (table 3).

\section{Stage II versus Stage IV}

CEA, IL- 6 and IL-8 accurately differentiated between stage II and stage IV. The most effective was CEA (AUC $0.81,95 \% \mathrm{Cl} 0.67-0.95, p=0.001$ ) followed by IL-8 (AUC $0.80,95 \% \mathrm{Cl} 0.66-0.93, \mathrm{p}=0.001$ ) then IL-6 (AUC 0.75, $95 \% \mathrm{Cl} 0.58-0-.91, \mathrm{p}=0.007$ ) (fig. 2). Interleukin-8 was therefore identified as the best cytokine to combine with CEA. The optimal cut off values were $4.4 \mu \mathrm{g} / \mathrm{L}$ for CEA (sensitivity 0.84 , specificity 0.54 ) and $6.6 \mathrm{pg} / \mathrm{mL}$ for IL-8 (sensitivity 0.84 , specificity 0.59 ). Patients with a low CEA and low IL-8 were assigned a combined
Table 3 - Receiver Operator Characteristic curve analysis

\begin{tabular}{lcccc}
\hline & \multicolumn{2}{c}{ Control v Stage II } & \multicolumn{2}{c}{ Control v Stage IV } \\
\hline & AUC & $p$ & AUC & $p$ \\
\hdashline IL6 & 0.55 & 0.619 & 0.78 & 0.003 \\
\hdashline IL8 & 0.68 & 0.057 & 0.92 & $<0.0001$ \\
\hdashline CEA & 0.75 & 0.008 & 0.92 & $<0.0001$ \\
\hline
\end{tabular}

CEA-IL8 score of ' 0 ' and patients with a high CEA and/or IL-8 were assigned a score of ' 1 '.

Of forty-one patients with colorectal cancer, 17 (41.4\%) had a 'low' and 24 (58.5\%) had a 'high' CEA. Stage IV disease was present in $4 / 17$ (23.5\%) with low CEA compared to $15 / 24$ (62.5\%) of those with a high CEA. Nine of the 41 patients with CRC (22.0\%) had a
A: Interleukin-6

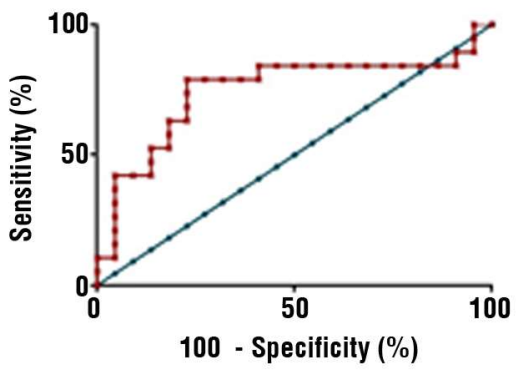

B: Interleukin-8

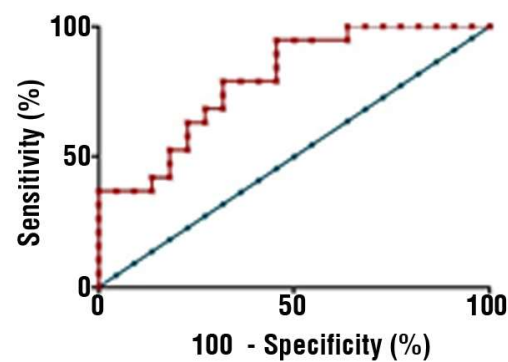

C: CEA

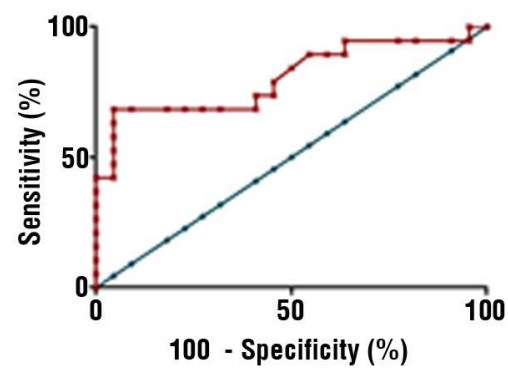

Figure 2 - Receiver Operator Characteristic curves (red) evaluating ability of IL-6 (A), IL-8 (B) and CEA (C) to differentiate between Stage II and Stage IV. Reference line shown in blue 
CEA-IL8 score of ' 0 ' and 32 (78.0\%) had a score of ' 1 '. No significant associations were observed between the CEA-IL8 score and any of the demographic variables measured. Stage IV disease was present in $1 / 9(11.1 \%)$ of patients with a CEA-IL8 of ' 0 ' compared to $18 / 32$ (56.3\%) of those with a score of ' 1 '.

The IL8-CEA score was more effective in discriminating between stage IV and stage II CRC (OR 10.3, 95\% Cl 1.192.2, $\mathrm{p}=0.01$ ) compared with CEA alone (OR $5.4,95 \% \mathrm{Cl}$ $1.3-27.8, \mathrm{p}=0.01$ ).

\section{DISCUSSION}

In this study of sixty prospectively-enrolled participants, we found CEA to discriminate between healthy controls and those with stage II disease, whereas inflammatory markers IL-6 and IL-8 did not. We found all three markers to vary significantly between those with stage II and stage IV disease. IL-8 was the more effective inflammatory marker, and when combined with CEA, the resulting score outperformed CEA alone in discriminating between stage II and stage IV disease.

An association between raised serum CEA and the presence of early-stage CRC has been observed in other studies (22). However, as demonstrated in a metaanalysis by Liu et al, poor sensitivity limits its application as a screening or diagnostic test (23). Although the existing data is much sparser for interleukins, a pooled analysis of five case-control studies including 668 CRC patients and 374 controls showed IL-8 to have moderate diagnostic accuracy (23). This was not corroborated by the findings of this study and while this may have been partly due to the small sample size, we were not led to evaluate a score combining CEA with an inflammatory cytokine in the discrimination between healthy controls and stage II disease.

Our finding that CEA, IL-6 and IL-8 effectively discriminated between stages II and IV disease supports the findings of others. In their study of 75 patients, Mahboob et al utilised Proseek and bioplex immunoassays and identified CEA, IL- 6 and IL- 8 as amongst the most stage-specific cytokines from a panel of 92 protein biomarkers (24). Paczel et al's recent study of 59 CRC patients found a raised CEA $(p<0.005)$ and IL-8 $(p=0.004)$ to be significantly associated with the presence of distant metastases (15). Liu et al's study of 30 patients did not find IL-6 or IL-8 to differ significantly by TNM stage although they did not analyse stage IV disease separately but compared stages 0 and I with stages II, III and IV (25).

To our knowledge, a combined IL8-CEA score, such as that utilised in the present study, has not been previously evaluated as a marker of metastatic CRC. However, the approach of combining CEA with circulating inflammatory biomarkers has shown some promise in diagnostic studies. Li et al found that combining platelet-lymphocyte ratio (PLR) or lymphocyte-monocyte ratio (LMR) with CEA improved diagnostic efficacy for CRC (19), whereas Tang et al's study of 138 patients with non small cell lung carcinoma (NSCLC) found that the sensitivity of tumour markers including CEA in the diagnosis of NSCLC was improved by combining it with IL-6 (26).

Experience in the clinical setting suggests the CEA value to be an indicator of disease burden. This is apparent through its more established roles in post-operative surveillance for CRC recurrence and in monitoring metastatic disease progression in those undergoing chemotherapy (27). In contrast to this, circulating pro-inflammatory cytokines, such as IL-8, levels are likely to reflect a functional, host-response to cancer $(11,12,14)$. IL-8 is a chemokine produced primarily by macrophages resulting in neutrophil chemo-attraction into the tumour microenvironment (11). Furthermore, animal studies have shown that exposure to IL-8 may increase the metastatic potential of colon cancer cells (28). In their experiments on mice using a circulating tumour cell (CTC) model, Kim et al found that tumour-derived IL-8 acted as a chemoattractant of tumour cells to the primary tumour and metastatic sites $(29,30)$. Our observation that combining IL-8 with CEA improves upon the stagespecificity of CEA alone may reflect a more global assessment of both disease burden and host-response.

This study was limited by a small sample size which may, in turn, have been partly a result of strict eligibility criteria. Patients presenting as an emergency and with chronic inflammatory conditions and a recent history of taking anti-inflammatory or immunomodulatory medications were excluded to avoid confounding. The results of this study may therefore not be generalizable to these patient sub-groups. While the findings of this study strengthen the approach of combining tumour markers with inflammatory biomarkers as a proof-of-principle, further validation studies are necessary in larger samples. Additionally, we were unable to control for histopathological features such as $\mathrm{T}$ stage, $\mathrm{N}$ stage and lympho-vascular invasion. This was not possible as most patients in the stage IV group did not undergo surgical resection of their primary tumour, but were instead treated with palliative chemotherapy. Limiting patients in the stage IV group to those undergoing resection of their primary tumour would exclude a majority of patients with 
disseminated disease and introduce an unacceptable likelihood of bias.

\section{CONCLUSION}

Circulating inflammatory cytokines IL-6 and IL-8 were not found to be effective discriminators between healthy controls and early-stage CRC. However, both biomarkers were effective in discriminating stage IV from stage II disease; IL-8 more so than IL-6. Combining CEA with IL-8 resulted in a score that outperformed CEA alone in discriminating between stage II and stage IV disease. Although these findings support the principle of combining CEA with IL-8 to accurately stage patients, larger validation studies are required. A combined IL8CEA score may add value to radiological pre-operative staging.

\section{Author contributions}

(1) Conception and design: PL, ED

(2) Administrative support: AG, SM, KD

(3) Provision of study material or patients: AG, SM, $\mathrm{AH}, \mathrm{GK}, \mathrm{AS}, \mathrm{ED}$

(4) Collection and assembly of data: AG, SM, KD, $\mathrm{AH}, \mathrm{GK}$

(5) Data analysis and interpretation: AG, PL, ED

(6) Manuscript writing: All authors

(7) Final approval of manuscript: All authors

\section{Acknowledgements}

The research was funded through awards by the Surgical Research Trust and Phil \& Teds. The authors would also like to thank Sara Farrant, Colorectal nurse specialist and Philippa Cashin, laboratory technician.

\section{Conflicts of Interest}

The authors all declare that they have no conflict of interest.

\section{Informed consent}

All procedures followed were in accordance with the ethical standards of the responsible committee on human experimentation (institutional and national) and with the Helsinki Declaration of 1975, as revised in 200 (5). Informed consent was obtained from all patients for being included in the study.

\section{REFERENCES}

1. Ferlay J, Soerjomataram I, Dikshit R, Eser S, Mathers C, Rebelo M, et al., Cancer incidence and mortality worldwide: sources, methods and major patterns in GLOBOCAN 2012. Int J Cancer. 2015;136(5): E359-86. Epub 2014 Oct 9.

2. Siegel RL, Miller KD, Fedewa SA, Ahnen DJ, Meester RGS, Barzi A, et al. Colorectal cancer statistics, 2017. CA Cancer J Clin. 2017; 67(3):177-193.

3. Lee JH, Lee SW. The roles of carcinoembryonic antigen in liver metastasis and therapeutic approaches. Gastroenterol Res Pract. 2017:2017:7521987.

4. Duffy MJ. Carcinoembryonic antigen as a marker for colorectal cancer: is it clinically useful? Clin Chem. 2001:47(4):624-30.

5. Compton C, Fenoglio-Preiser CM, Pettigrew N, Fielding LP. American Joint Committee on Cancer prognostic factors consensus conference. Cancer. 2000;88(7):1739-57.

6. Thirunavukarasu, P, Talati C, Munjal S, Attwood K, Edge SB, Francescutti $V$. Effect of incorporation of pretreatment serum carcinoembryonic antigen levels into AJCC staging for colon cancer on 5-year survival. JAMA Surg. 2015;150(8):747-755.

7. Spindler BA, Bergquist JR, Thiels CA, Habermann EB, Kelley SR, Larson DW, et al. Incorporation of CEA improves risk stratification in stage II colon cancer. J Gastrointest Surg. 2017;21(5):770-777.

8. Lasry A, Zinger A, Ben-Neriah Y. Inflammatory networks underlying colorectal cancer. Nat Immunol. 2016;17(3):230-40.

9. Park JH, Watt DG, Roxburgh CSD, Horgan PG, McMillan DC. Colorectal cancer, systemic inflammation, and outcome: staging the tumor and staging the host. Ann Surg. 2016;263(2):326-36.

10. Hanahan D, Weinberg RA. Hallmarks of cancer: the next generation. Cell. 2011;144(5):646-74.

11. Mager LF, Wasmer MH, Rau TT, Krebs P. Cytokine-Induced Modulation of Colorectal Cancer. Front Oncol. 2016:6:96.

12. McAllister SS, Weinberg RA. The tumour-induced systemic environment as a critical regulator of cancer progression and metastasis. Nat Cell Biol. 2014;16(8):717-27.

13. Tuomisto AE, Mäkinen MJ, Väyrynen JP. Systemic inflammation in colorectal cancer: Underlying factors, effects, and prognostic significance. World J Gastroenterol. 2019;25(31):4383-4404.

14. West NR, McCuaig S, Franchini F, Powrie F. Emerging cytokine networks in colorectal cancer. Nat Rev Immunol. 2015;15(10):615-29.

15. Paczek S, Łukaszewicz-Zajac M, Gryko M, Mroczko P, KulczyńskaPrzybik A, Mroczko B. CXCL-8 in Preoperative Colorectal Cancer Patients: Significance for Diagnosis and Cancer Progression. Int J Mol Sci. 2020;21(6):2040.

16. Gunawardene, A., E. Dennett, and P. Larsen. Prognostic value of multiple cytokine analysis in colorectal cancer: a systematic review. J Gastrointest Oncol. 2019;10(1):134-143.

17. Kim YW, Kim SK, Kim CS, Kim IY, Cho MY, Kim NK. Association of serum and intratumoral cytokine profiles with tumor stage and neutrophil lymphocyte ratio in colorectal cancer. Anticancer Res. 2014;34(7):3481-7.

18. Kantola T, Klintrup K, Väyrynen JP, Vornanen J, Bloigu R, Karhu T, et al. Stage-dependent alterations of the serum cytokine pattern in colorectal carcinoma. Br J Cancer. 2012;107(10):1729-36.

19. Li X, Guo D, Chu L, Huang Y, Zhang F, Li W et al. Potential diagnostic value of combining inflammatory cell ratios with carcinoembryonic Antigen For Colorectal Cancer. Cancer Manag Res. 2019;11:9631-9640.

20. Peng $H X$, Yang L, He BS, Pan $Y Q$, Ying $H Q$, Sun $H L$, et al. Combination of preoperative NLR, PLR and CEA could increase the diagnostic efficacy for I-III stage CRC. J Clin Lab Anal. 2017;31(5): e22075. Epub 2016 Sep 30.

21. Jessup JM, et al. Colon and Rectum, in AJCC Cancer Staging Manual, M.B. Amin, et al., Editors. 2017, Springer International Publishing AG: Cham, Switzerland. p. 252-274.

22. Palmqvist R, Engarțs $B$, Lindmark G, Hallmans G, Tavelin B, Nilsson $O$, et al. Prediagnostic levels of carcinoembryonic antigen and $C A$ 242 in colorectal cancer: a matched case-control study. Dis Colon Rectum. 2003:46(11):1538-44. 
23. Liu Z, Zhang Y, Niu Y, Li K, Liu X, Chen H, et al. A systematic review and meta-analysis of diagnostic and prognostic serum biomarkers of colorectal cancer. PLoS One. 2014;9(8):e103910.

24. Mahboob S, Ahn SB, Cheruku HR, Cantor D, Rennel E, Fredriksson $S$, et al. A novel multiplexed immunoassay identifies CEA, IL-8 and prolactin as prospective markers for Dukes' stages $A D$ colorectal cancers. Clin Proteomics. 2015;12(1):10.

25. Liu H, Zhang Z, Tabuchi T, Wang S, Wang J. The role of proinflammatory cytokines and immune cells in colorectal carcinoma progression. Oncol Lett. 2013;5(4):1177-1182.

26. Tang H, Bai $Y$, Shen W, Wei $Y, X u$ M, Zhou X, et al. Clinical significance of combined detection of interleukin- 6 and tumour markers in lung cancer. Autoimmunity. 2018;51(4):191-198.

27. Duffy M, Lamerz R, Haglund C, Nicolini A, Kalousová M, Holubec L, et al., Tumor markers in colorectal cancer, gastric cancer and gastrointestinal stromal cancers: European group on tumor markers 2014 guidelines update. Int J Cancer. 2014;134(11):2513-22. Epub 2013 Aug 27.

28. Lee YS, Choi I, Ning Y, Kim NY, Khatchadourian V, Yang D, et al. Interleukin-8 and its receptor CXCR2 in the tumour microenvironment promote colon cancer growth, progression and metastasis. $\mathrm{Br}$ J Cancer. 2012;106(11):1833-41.

29. Bie Y, Ge W, Yang Z, Cheng X, Zhao Z, Li S, et al. The crucial role of CXCL8 and its receptors in colorectal liver metastasis. Dis Markers. 2019;2019:8023460

30. Kim MY, Oskarsson T, Acharyya S, Nguyen DX, Zhang XHF, Norton $\mathrm{L}$, et al. Tumor self-seeding by circulating cancer cells. Cell. 2009; 139(7):1315-26 